\title{
Fractional-Order Controller Design for Oscillatory Fractional Time-Delay Systems Based on the Numerical Inverse Laplace Transform Algorithms
}

\author{
Lu Liu, Feng Pan, and Dingyu Xue \\ College of Information Science and Engineering, Northeastern University, Shenyang 110819, China \\ Correspondence should be addressed to Feng Pan; panfeng@ise.neu.edu.cn
}

Received 19 October 2014; Revised 24 February 2015; Accepted 8 March 2015

Academic Editor: Rosana Rodriguez-Lopez

Copyright @ $2015 \mathrm{Lu}$ Liu et al. This is an open access article distributed under the Creative Commons Attribution License, which permits unrestricted use, distribution, and reproduction in any medium, provided the original work is properly cited.

Fractional-order time-delay system is thought to be a kind of oscillatory complex system which could not be controlled efficaciously so far because it does not have an analytical solution when using inverse Laplace transform. In this paper, a type of fractional-order controller based on numerical inverse Laplace transform algorithm INVLAP was proposed for the mentioned systems by searching for the optimal controller parameters with the objective function of ITAE index due to the verified nature that fractional-order controllers were the best means of controlling fractional-order systems. Simulations of step unit tracking and load-disturbance responses of the proposed fractional-order optimal $P I^{\lambda} D^{\mu}$ controller (FOPID) and corresponding conventional optimal PID (OPID) controller have been done on three typical kinds of fractional time-delay system with different ratio between time delay $(L)$ and time constant $(T)$ and a complex high-order fractional time delay system to verify the availability of the presented control method.

\section{Instruction}

Fractional-order calculus, strictly speaking, nonintegerorder calculus, as a part of derivatives mathematics, which is dealing with arbitrary order derivatives, attracted more and more attention and developed extensively in numerous fields in the past few years. It costs a fairly long period of time on the development of research of fractional calculus applying on control systems from the 1960s, and until the end of the 20th century, a number of remarkable achievements have been obtained by some researchers, for instance, that the CRONE control principle was proposed by Oustaloup et al. (1995) [1]; Matignon (1996) investigated the stability, controllability, and observability of fractional-order systems $[2,3]$; one of the notable landmarks on the designing of fractional-order PID controller was achieved by Podlubny (1998) that he extended the traditional notion of PID controller into more flexible structure and made the number of gain variables of PID controllers increased from 3 to 5 with two extra fractional differential and integral orders and it was more suited for the control of noninteger-order systems [4]. In recent years, fractional calculus has been applied increasingly in various fields such as mathematics, engineering, biomedicine, and science [5-8]. At the same time, an increasing number of dynamic complicated systems and physical phenomena were described by fractional-order models to represent natural phenomenon in a better way $[9,10]$. Meanwhile, numerous ways of fractional-order controller design emerged with the rapid development of fractional-order models. Valério and da Costa (2006) used Ziegler-Nichols-type rule while designing a fractional PID controller [11]; Monje et al. (2004) proposed some tuning rules for robustness to plant uncertainties on fractional PID controllers [12]; Sadati et al. (2007) have shown a PSO based approach on fractional controller designing for SISO or MIMO systems [13]. A RBF neural network based FOPID controller which trained a large amount of controller and system parameters by frequency domain robust tuning approach was designed by Chen et al. (2010) [14].

However, among the existing fractional controller tuning methods, few of them have been used on fractional plants with time delay because of the inherent complexities of the systems and the difficulty on inverse Laplace transforms 
calculating. In this paper, a novel FOPID tuning method based on the numerical inverse Laplace transform algorithms is proposed for the time-delay fractional systems, and a set of noninteger-order time-delay (NIOTD) systems [15] were tested for universality in the simulation chapter. Pan et al. (2011) [16] have shown that NIOTD could represent many higher order systems succinctly and precisely; hence this kind of simulation would give rise to some meaningful conclusions. The application of numerical inverse Laplace transform algorithms in fractional calculus has been researched by Sheng et al. (2011) [17], analytical results of three kinds of numerical inverse Laplace transform algorithms that is INVLAP, Gavsteh, and NILT have been compared and it is proved that the INVLAP algorithm used in this paper was effective and reliable for fractional-order differential equations and offered a simple and convenient way to solve the controller design problem for the complicated fractionalorder systems with time delay.

Laplace transform is considered to be available in solving integer-order and some ordinary fractional-order differential $[4,18]$, and inverse Laplace transform is thought to be a significant but tough step in using it to solve differential equations. For a complex system, for example, fractional-order time-delay system, it is unable to accomplish the inverse Laplace transform analytically by using its definition or consulting the Laplace transform table [19]. Therefore, the numerical inverse Laplace transform algorithm is always used in solving this kind of problems and finding a numerical result. In this paper, numerical inverse Laplace transform algorithm was applied in designing a FOPID controller for NIOTD systems and its availability has been verified by simulation results.

The rest part of this paper is divided as follows: as a starting point, numerical inverse Laplace transform algorithm INVLAP and fractional calculus are introduced briefly in Section 2. And then in Section 3, tuning method of fractional-order controller, performance index, and optimization means are presented. After that, a series of simulation illustrations are shown in Section 4 to demonstrate the effectiveness and robustness of the designed FOPID controller compared with the corresponding OPID controller. Finally, conclusions are drawn in Section 5.

\section{Preliminaries}

2.1. Numerical Inverse Laplace Transform Algorithm INVLAP. Inverse Laplace transform is considered to be a vital but difficult part in using Laplace transform to solve fractionalorder differential equations, and even some of the differential equations do not have an analytical solution at all, for example, fractional-order time-delay systems. That is the reason that there have been few controllers designed for this kind of systems; however, actually the mentioned systems are meaningful and representative in control field; that is also why numerical inverse Laplace transform algorithm INVLAP was applied in this paper [20].
The inverse Laplace transform function is of the form

$$
f(t)=L^{-1}\{F(s)\}=\frac{1}{2 \pi i} \int_{\sigma-i \infty}^{\sigma+i \infty} F(s) e^{s t} d s,
$$

where $s=\sigma+j w$.

Some assumptions were made to be fulfilled:

(i) $\lim _{|s| \rightarrow \infty} F(s)=0$;

(ii) $F(s)$ is regular and $\operatorname{Re}\{s\}>0$;

(iii) $F\left(s^{*}\right)=F^{*}(s)$, where $*$ means complex conjugation.

Then, the exponential function could be simplified by

$$
\begin{aligned}
e^{s t} & \cong E(s t, a)=\frac{e^{a}}{2 \sinh (a-s t)} \\
& =\frac{e^{a}}{e^{a} e^{-s t}-e^{-a} e^{s t}}=\frac{e^{a}}{1-e^{-2 a} e^{2 s t}},
\end{aligned}
$$

where $\left|e^{-2 a} e^{2 s t}\right| \ll 1$ when $a>\sigma t$ and then $1 /\left(1-e^{-2 a} e^{2 s t}\right)$ could be expanded as a convergent MacLaurin series as

$$
E(s t, a)=e^{s t}+\sum_{n=1}^{\infty} e^{-2 n a} e^{(2 n+1) s t} .
$$

The approximation error of the exponential term can be represented by the sum term in (3) and it may be suppressed by the selected value of parameter $a$ that

$$
\begin{aligned}
f(t) \approx & f(t, a) \\
= & \frac{1}{2 \pi i} \int_{\sigma-i \infty}^{\sigma+i \infty} F(s) E(s t, a) d s \\
= & \frac{1}{2 \pi i} \int_{\sigma-i \infty}^{\sigma+i \infty} F(s) e^{s t} d s \\
& +\frac{1}{2 \pi i} \int_{\sigma-i \infty}^{\sigma+i \infty} F(s) \sum_{n=1}^{\infty} e^{-2 n a} e^{(2 n+1) s t} d s \\
= & f(t)+\sum_{n=1}^{\infty} e^{-2 n a} f[(2 n+1) t] \\
= & f(t)+e^{-2 a} f(3 t)+e^{-4 a} f(5 t) \cdots \\
= & f(t)+\varepsilon(t, a) .
\end{aligned}
$$

$\varepsilon(t, a)$ represents the error value of inverse transform and it could be reduced by changing the value of parameter a similarly. The function $E(s t, a)$ could be replaced by an infinite number of rational functions as [18]

$$
\frac{1}{\sinh z}=\frac{1}{z}+2 \pi \sum_{n=1}^{\infty} \frac{(-1)^{n}}{n^{2} \pi^{2}+z^{2}}
$$

Then, we achieved that

$$
E(s t, a)=e^{a}\left[\frac{1}{2(a-s t)}+(a-s t) \sum_{n=0}^{\infty} \frac{(-1)^{n}}{n^{2} \pi^{2}+(a-s t)^{2}}\right] .
$$


So,

$$
\begin{array}{r}
f(t, a)=\frac{e^{a}}{2 \pi i} \int_{\sigma-i \infty}^{\sigma+i \infty} F(s)\left[\frac{1}{2(a-s t)}+(a-s t)\right. \\
\left.\cdot \sum_{n=0}^{\infty} \frac{(-1)^{n}}{n^{2} \pi^{2}+(a-s t)^{2}}\right] d s .
\end{array}
$$

Then we substituted the sequence of summation by integration that

$$
f(t, a)=\frac{e^{a}}{2 \pi i}\left[\frac{1}{2} I_{0}+\sum_{n=1}^{\infty}(-1)^{n} I_{n}\right]
$$

where

$$
I_{n}=\int_{\sigma-i \infty}^{\sigma+i \infty} \frac{(a-s t) F(s)}{n^{2} \pi^{2}+(a-s t)^{2}} d s, \quad n=0,1,2, \ldots
$$

Assume that $G_{n}(s)=n^{2} \pi^{2}+(a-s t)^{2}$, so

$$
I_{n}=\int_{\sigma-i \infty}^{\sigma+i \infty} \frac{(a-s t) F(s)}{G_{n}(s)} d s=\int_{\sigma-i \infty}^{\sigma+i \infty} H_{n}(s) d s .
$$

The integration $I_{n}$ has been proved to be equal to the total value of the residua of $H_{n}(s)$ in [21]. Then, the roots of equation $G_{n}(s)=0$ are

$$
s_{1,2}=\frac{a \pm n \pi i}{t} .
$$
lated as

The path of the integration is easy to be closed and calcu-

$$
\begin{aligned}
I_{n} & =2 \pi i\left[\operatorname{res}_{s=s_{1}} H_{n}(s)+\underset{s=s_{2}}{\operatorname{res}} H_{n}(s)\right] \\
& =2 \pi i\left[\frac{F\left(s_{1}\right)}{G_{n}^{\prime}\left(s_{1}\right)}+\frac{F\left(s_{2}\right)}{G_{n}^{\prime}\left(s_{2}\right)}\right] .
\end{aligned}
$$

And obviously,

$$
\begin{aligned}
& I_{n}=2 \pi i\left[\frac{F\left(s_{1}\right)}{2 \pi t n}+\frac{F\left(s_{2}\right)}{2 \pi t n}\right]=\frac{2 \pi i \operatorname{Re}\{F((a+n \pi i) / t)\}}{t}, \\
& I_{0}=\frac{2 \pi i}{t} F\left(\frac{a}{t}\right) .
\end{aligned}
$$

Therefore, we got

$$
f(t, a)=\frac{e^{a}}{t}\left[\frac{1}{2} F\left(\frac{a}{t}\right)+\sum_{n=0}^{\infty}(-1)^{n} \operatorname{Re}\left\{F\left(\frac{a+i n \pi}{t}\right)\right\}\right] .
$$

There was also another form of expression which was described by the imaginary part of $F(s)$ corresponding to the real part expression in (14), and details could be found in [21].
2.2. Fractional Calculus. The fractional operator is defined as

$$
{ }_{a} D_{t}^{\alpha}= \begin{cases}\frac{d^{\alpha}}{d t^{\alpha}}, & R(\alpha)>0, \\ 1, & R(\alpha)=0, \\ \int_{a}^{t}(d \tau)^{(-\alpha)}, & R(\alpha)<0 .\end{cases}
$$

The Caputo definition is defined as

$$
{ }_{a} D_{t}^{\alpha}=\frac{1}{\Gamma(m-\alpha)} \int_{a}^{t} \frac{f^{m}(\tau)}{(t-\tau)^{\alpha-m+1}} d \tau,
$$

where $m$ donates the integer part of $\alpha, m=[\alpha]+1$, and $\Gamma(\cdot)$ is Euler's gamma function:

$$
\Gamma(s)=\int_{0}^{\infty} t^{s-1} e^{-t} d t
$$

in which if $s$ is an integer, it is obtained that

$$
\Gamma(s+1)=s ! .
$$

Then, the Laplace transform of the fractional-order operator is expressed by

$$
L\left[D^{\alpha} f(t)\right]=s^{\alpha} F(s)-\sum_{k=0}^{m-1} s^{k}\left[D^{\alpha-k-1} f(t)\right]_{t=0},
$$

where $m-1 \leq \alpha<m$. More fractional-order Laplace transforms could be found in Appendix in [19].

\section{Controller Design}

3.1. Fractional-Order $P I^{\lambda} D^{\mu}$ Controller. The integral-differential equation defined the control action of a $P I^{\lambda} D^{\mu}$ controller by

$$
u(t)=K_{p} e(t)+K_{i} D^{-\lambda} e(t)+K_{d} D^{\mu} e(t) .
$$

Then the transfer function of the $P I^{\lambda} D^{\mu}$ controller could be expressed as

$$
G_{c}(s)=K_{p}+\frac{K_{i}}{s^{\lambda}}+K_{d} s^{\mu}=k \frac{\left(s / \omega_{f}\right)^{\lambda+\mu}+s \delta_{f} s^{\lambda} / \omega_{f}+1}{s^{\lambda}} .
$$

$K_{p}, K_{i}$, and $K_{d}$ have the same meaning as the gains of classical integer-order PID controller are differential and integral orders, they could be arbitrary and lead to a more extensive scale of tuning; however, the regulating processes turn into more complex at the same time. When $\lambda=\mu=1$, it presents the traditional integer-order PID controller. Figure 1 shows the different tuning scale of PID and $P I^{\lambda} D^{\mu}$ controllers intuitively [22]. $P I^{\lambda} D^{\mu}$ controller extends the four control points of the traditional PID controller to the quarter-plane range of control points by changing the values of $\lambda$ and $\mu$. And the search scope of $\lambda, \mu$ in this paper is $(0,2)$. 

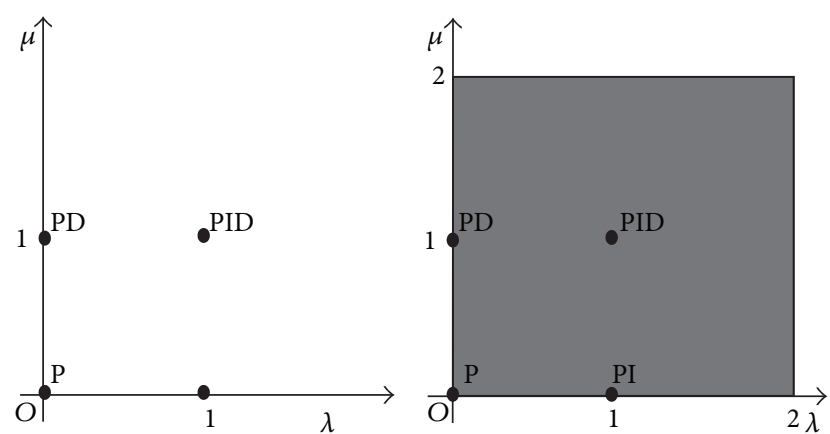

FIgURE 1: Tuning scale of PID controller and $P I^{\lambda} D^{\mu}$ controller.

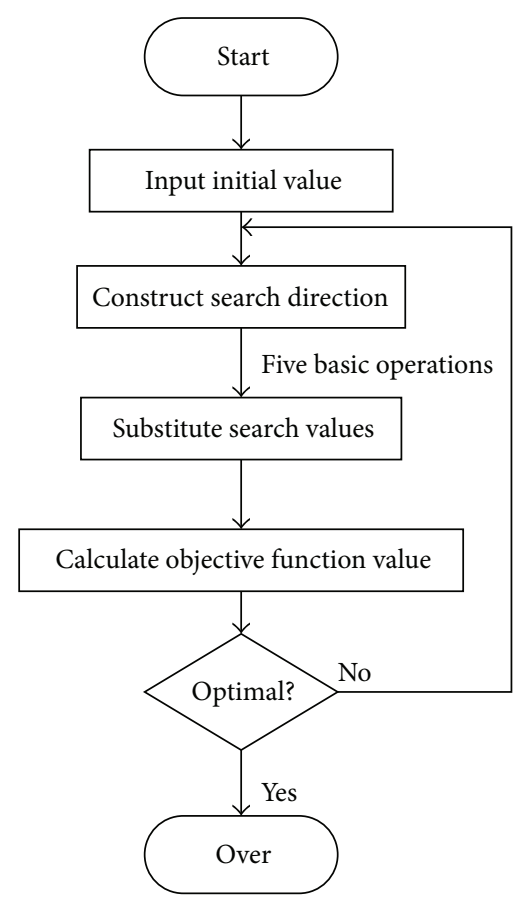

FIGURE 2: Flow chart of NM method optimization process.

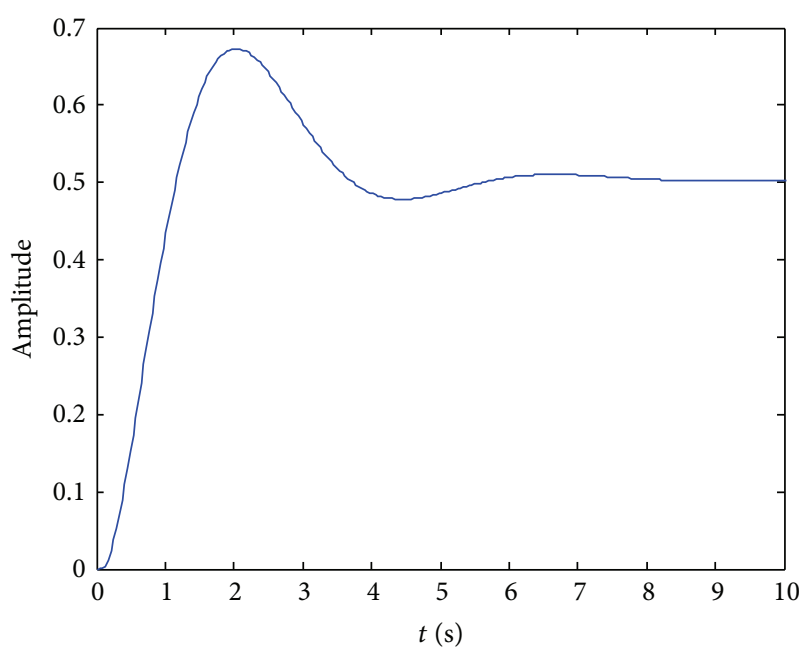

FIGURE 3: Numerical set-point tracking characteristics of $G_{1}(s)$.

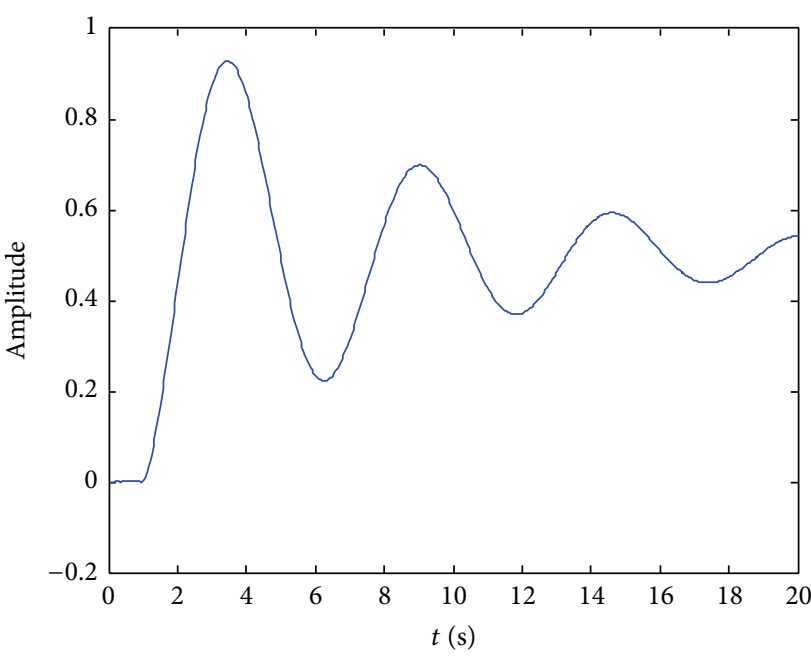

FIGURE 4: Numerical set-point tracking characteristics of $G_{2}(s)$.

3.2. Performance Criterion. Different performance criterions like ITAE, ISCO, ITSE, ISTES, and so on have been applied on fractional-order systems and compared with step set-point tracking and load-disturbance performance [23] and each integral performance criterion has different advantages when applied on designing of control systems. Among them, the ITAE index is a tradeoff between the absolute error and the settling time, which cannot be achieved by other criterions, and it has been the most commonly used one in fractionalorder controller design. So, the objective function used in this paper is given as

$$
J=\int_{0}^{\infty} t|e(t)| d t
$$

3.3. Optimization. The optimization method used in this paper is the Nelder-Mead (NM) simplex optimization method which is proposed by J. A. Nelder and R. Mead $[24,25]$. The basic thought of NM method is constructing a cursory search direction and implementing its basic five operations (reflection, expansion, compression inside, compression outside, and compression entirety) to search and substitute for the peak synchronously. The search direction of NM method is acquired by the assessment of objective function rather than direction gradient, which is the center of area shaped by all the peaks except for the worst one. We gave the initial value of the search points at first, and the values will be replaced by some better ones according to the value of objection function through the above five basic operations. Our expectant optimal $P I^{\lambda} D^{\mu}$ controller parameters are achieved by minimizing the ITAE performance index which was mentioned in above section as the objective function. The optimization process is shown as Figure 2.

\section{Simulation Results Validation}

4.1. Numerical Results of Typical Oscillatory Fractional-Order Time-Delay Systems. In the last few years, noninteger-order time-delay (NIOTD) system has been proved to be the 


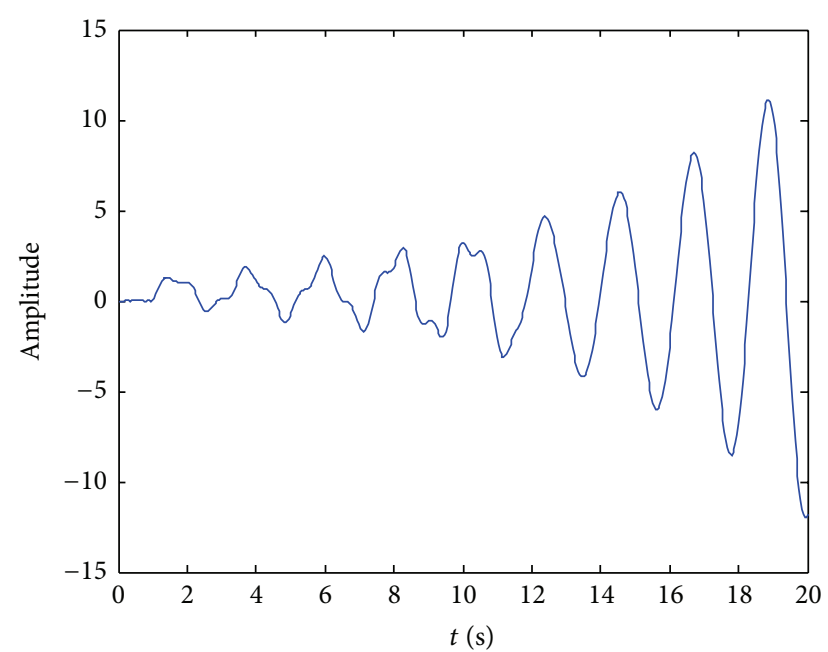

FIgURE 5: Numerical set-point tracking characteristics of $G_{3}(s)$.

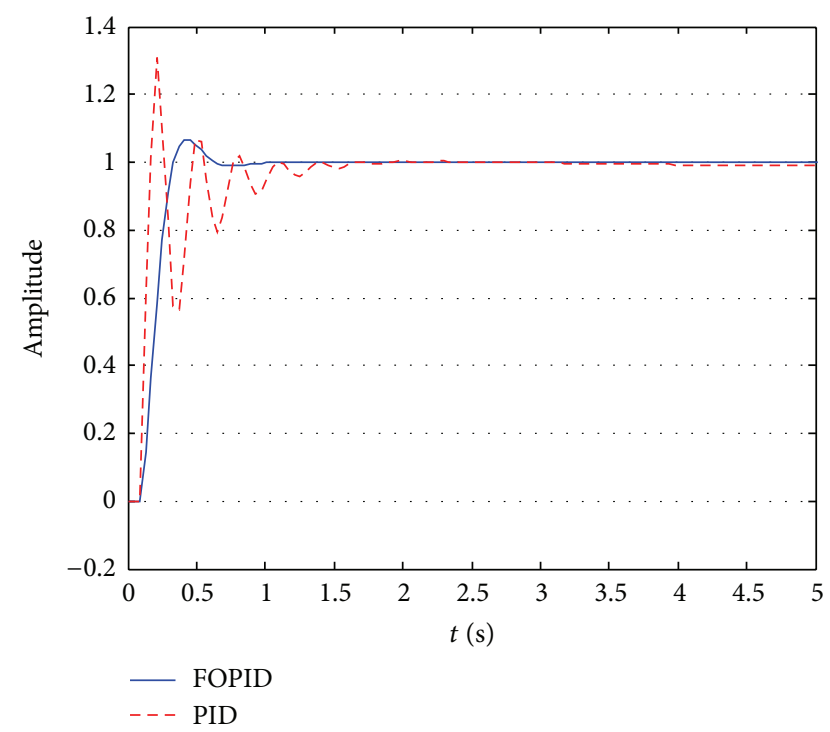

FIGURE 6: Set-point tracking control performance comparison of $G_{1}(s)$.

precisely and succinctly transformation model of many highorder complicated systems [16], and it has also been shown that fractional-order controllers were the best kind of controllers corresponding to such systems [15]. However, scarcely any fractional-order controller was designed for this kind of systems due to the fact that it did not have an analytical solution which was because of the internal complexity of time-delay $e^{-L s}$. The fractional-order delay system can be broadly represented as

$$
G(s)=\frac{K}{T s^{\alpha}+1} e^{-L s}
$$

And in consideration of universality, three kinds of NIOTD objective systems with different proportion of

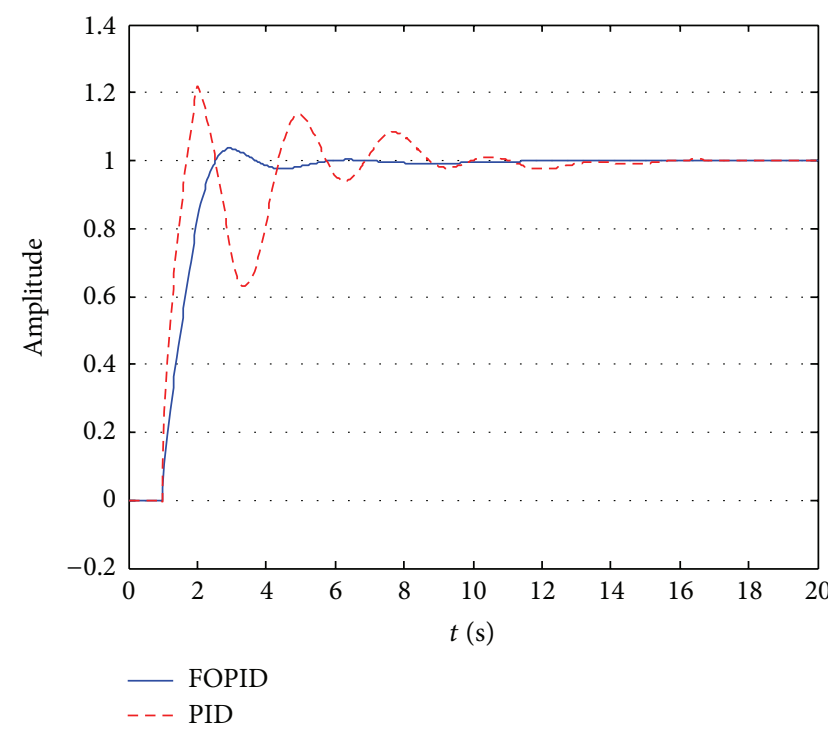

FIGURE 7: Set-pointing tracking control performance comparison of $G_{2}(s)$.

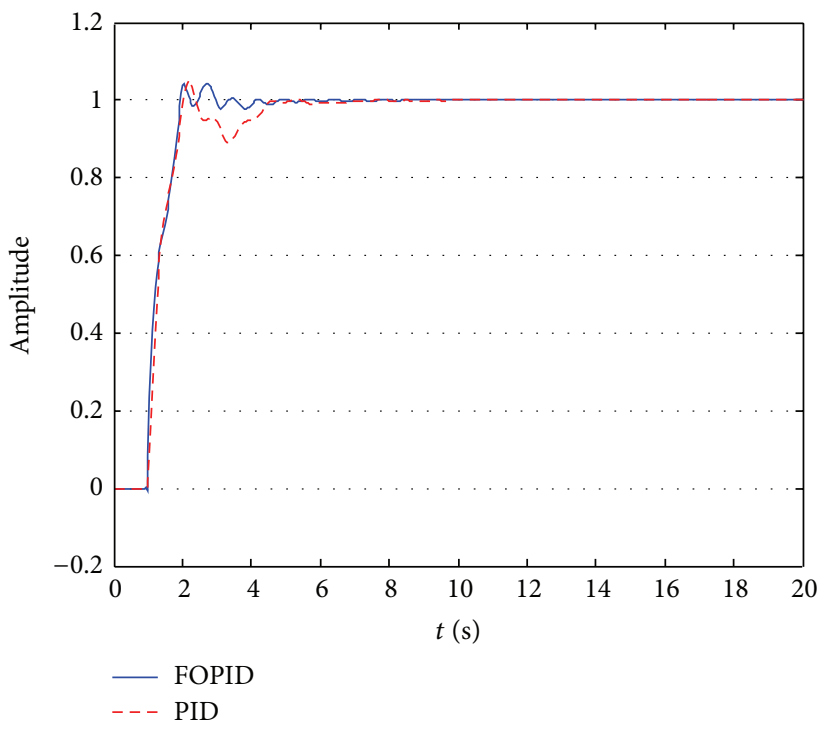

FIGURE 8: Set-pointing tracking control performance comparison of $G_{3}(s)$.

parameters, $L$, respectively, $T>L, T \approx L, T<L$, were tested [16]:

$$
\begin{aligned}
& G_{1}(s)=\frac{1}{1.11 s^{1.5}+1} e^{-0.105 s}, \\
& G_{2}(s)=\frac{1}{1.5 s^{1.5}+1} e^{-s}, \\
& G_{3}(s)=\frac{1}{0.004 s^{1.5}+1} e^{-s} .
\end{aligned}
$$

In this paper, numerical inverse Laplace transform algorithm INVLAP was applied to describe the tested complicated system. Then the initial numerical set-point tracking 


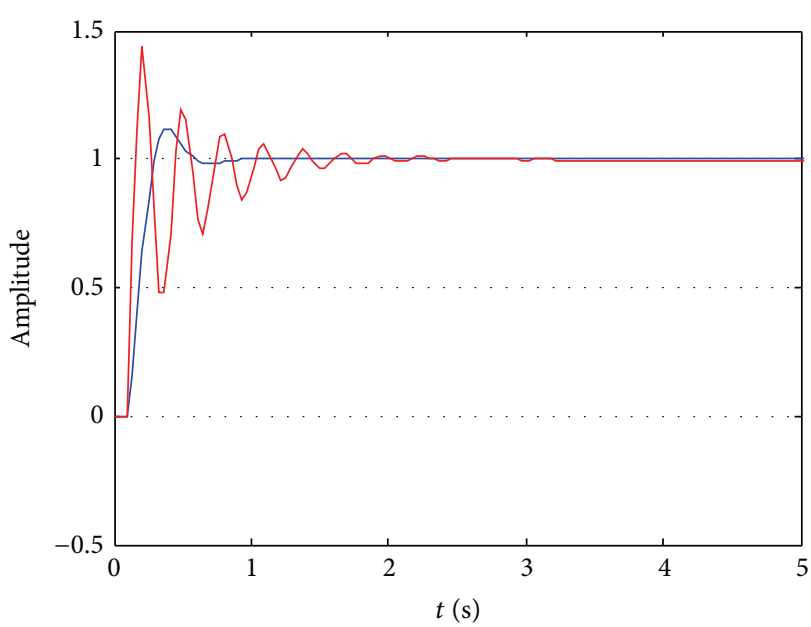

(a)

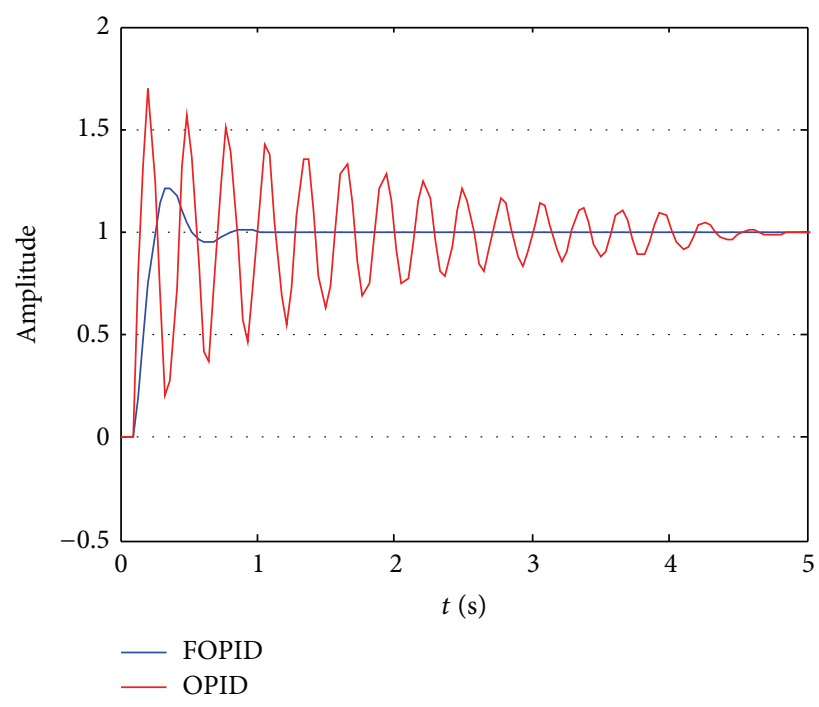

(c)

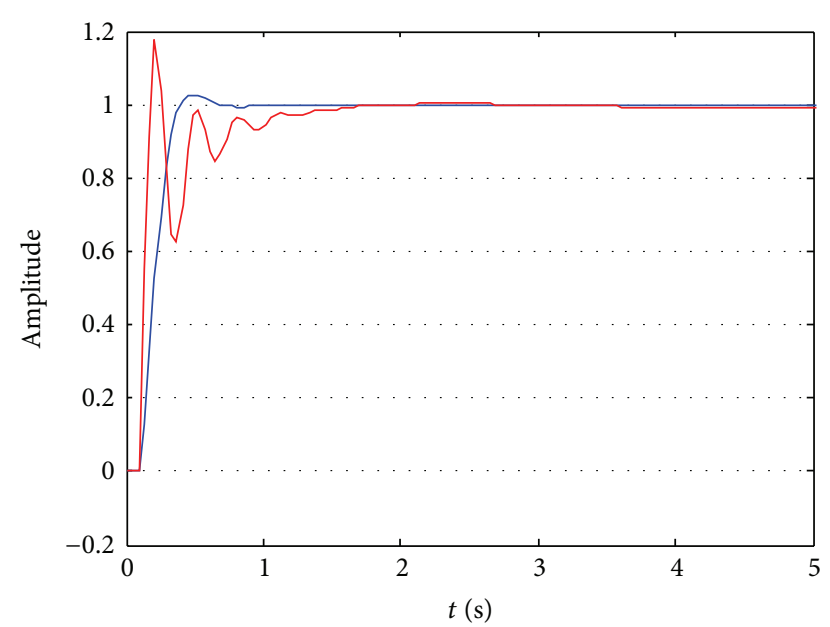

(b)

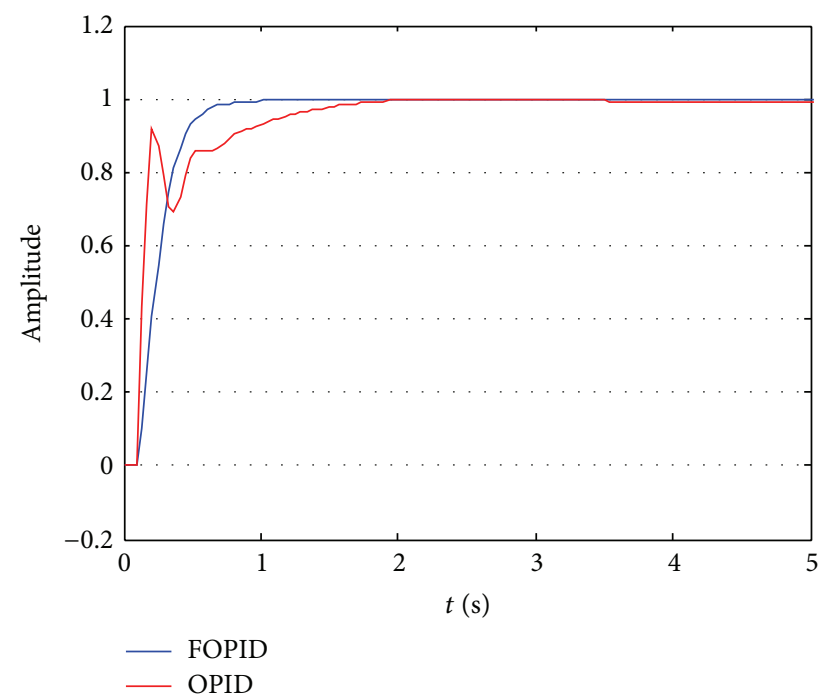

(d)

FIGURE 9: Load-disturbance response of $G_{1}(s)$.

characteristics of the three plants (24) could be achieved in Figures 3-5.

It has been shown in Figures 3-5 that $G_{1}(s)$ is almost convergent and $G_{2}(s)$ is oscillatory and may be convergent with a long accommodation time, but $G_{3}(s)$ is emanative; hence, a suited controller is necessary for each of them.

4.2. Comparative Performance of Different Controllers. In this section, a FOPID controller based on the tuning methods proposed in Section 3 and an OPID controller based on ITAE index are tuned, respectively, for $G_{1}(s), G_{2}(s)$, and $G_{3}(s)$ in Section 4.1. The controller parameters are listed in Table 1, comparative control performance of step set-point tracking is shown in Figures 6-8, and Figures 9-11 are comparisons of control performance with $\pm 10 \%, \pm 30 \%$ load-disturbance.

It is obviously to be seen that, comparing with the step input using OPID controller in Figures 6 and 7, the overshoots and accommodation time using FOPID controller are much smaller. In Figure 8, there is no much difference between the control performance of FOPID and OPID controllers; however, FOPID controller still holds superiority with accommodation time, and this phenomenon may be caused by the internal complexity and oscillatory property of the original system.

In order to show more potential and robustness of the presented FOPID controller, the disturbance rejection responses of FOPID and OPID controllers mentioned above are illustrated with $\pm 10 \%, \pm 30 \%$ load-disturbance in Figures 9-11. It is similar to the step set-point tracking responses; the disturbance rejection responses of $G_{1}(s)$ and $G_{2}(s)$ in Figures 9 and 10 show that FOPID controllers still perform well with satisfactory overshoots, rising time, and accommodation time, but the performance of OPID controllers is difficult to be accepted with high overshoots, long accommodation time, and even oscillate tempestuously. And in Figure 11, FOPID controller still shows more flexibility than OPID 
TABLE 1: Parameters of controllers.

\begin{tabular}{|c|c|c|c|c|c|c|c|}
\hline Control plant & Type of controller & $k_{p}$ & $k_{i}$ & $k_{d}$ & $\lambda$ & $\mu$ & $J_{\min }$ \\
\hline \multirow{2}{*}{$G_{1}(s)$} & FOPID & 0.3810 & 5.1988 & 5.5785 & 1.0006 & 0.5299 & 0.0081 \\
\hline & OPID & 4.0602 & 3.7491 & 4.3236 & - & - & 0.0535 \\
\hline \multirow{2}{*}{$G_{2}(s)$} & FOPID & 0.5590 & 0.6021 & 0.8613 & 1.0128 & 0.8845 & 0.4643 \\
\hline & OPID & 0.3072 & 0.0383 & 0.7511 & - & - & 1.0918 \\
\hline \multirow{2}{*}{$G_{3}(s)$} & FOPID & 0.1462 & 0.7339 & 0.1299 & 0.9982 & 0.2328 & 0.2667 \\
\hline & OPID & 0.3347 & 0.0063 & 0.6674 & - & - & 0.2701 \\
\hline
\end{tabular}

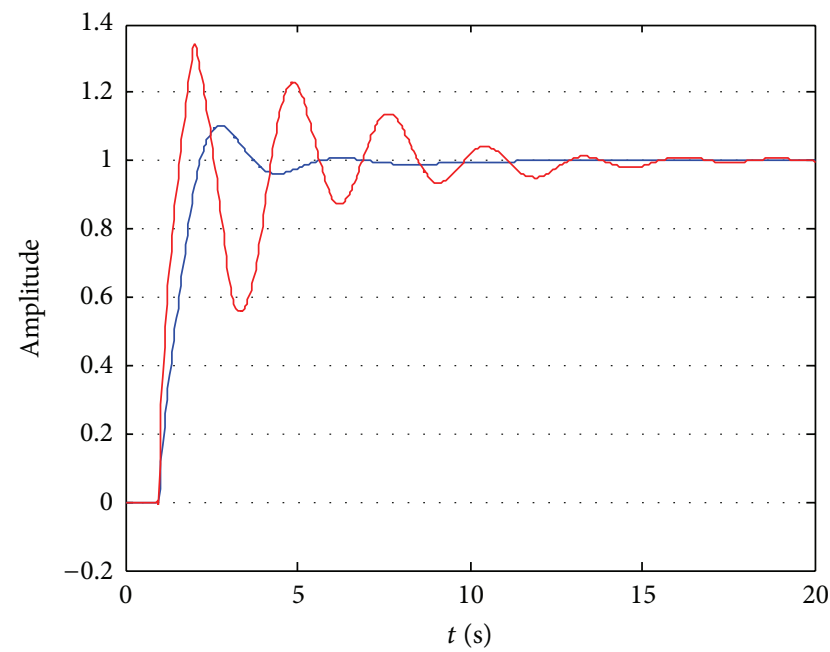

(a) $110 \%$ load-disturbance

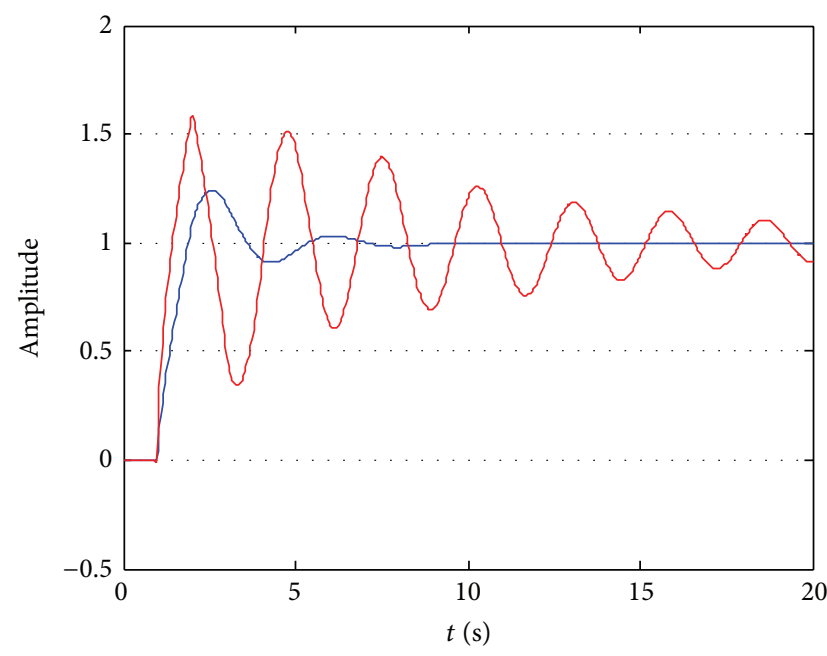

- FOPID

(c) $130 \%$ load-disturbance

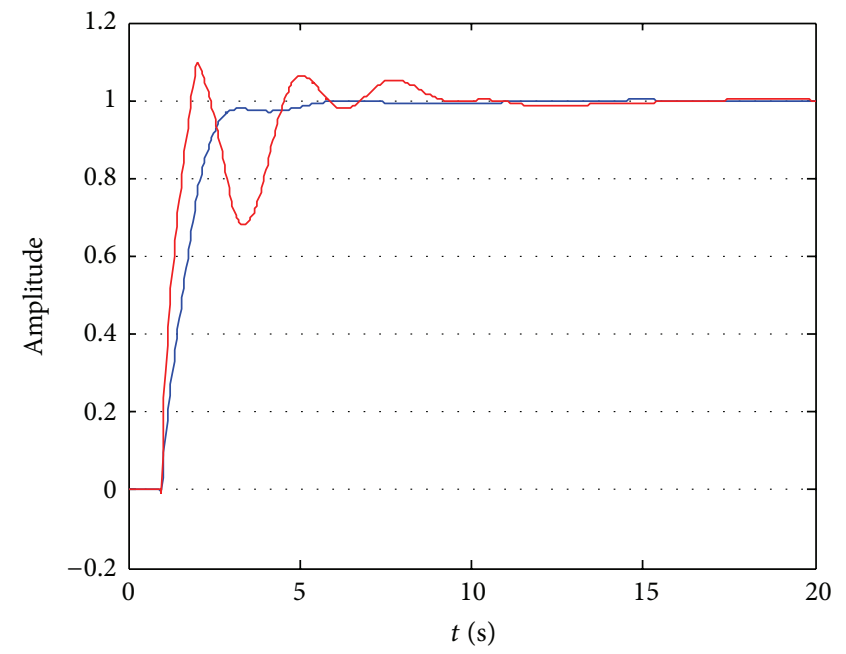

(b) $90 \%$ load-disturbance

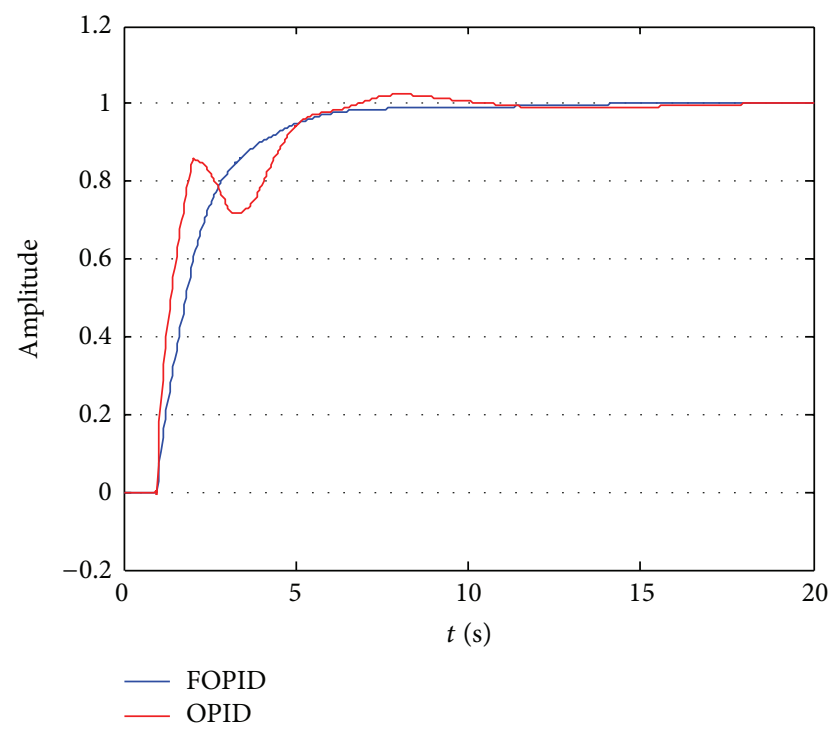

(d) 70\% load-disturbance

Figure 10: Load-disturbance response of $G_{2}(s)$. 


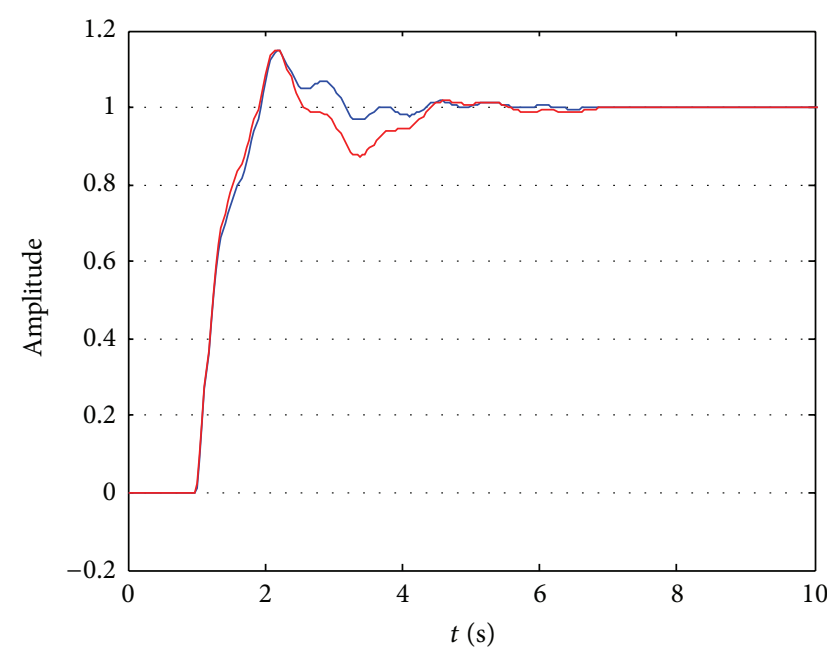

(a) 110\% load-disturbance

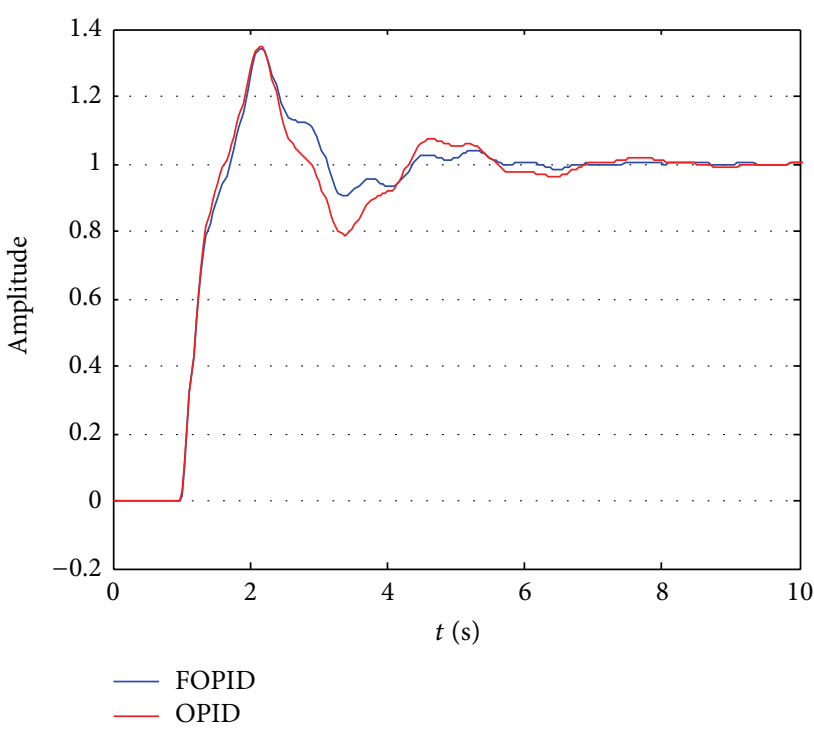

(c) $130 \%$ load-disturbance

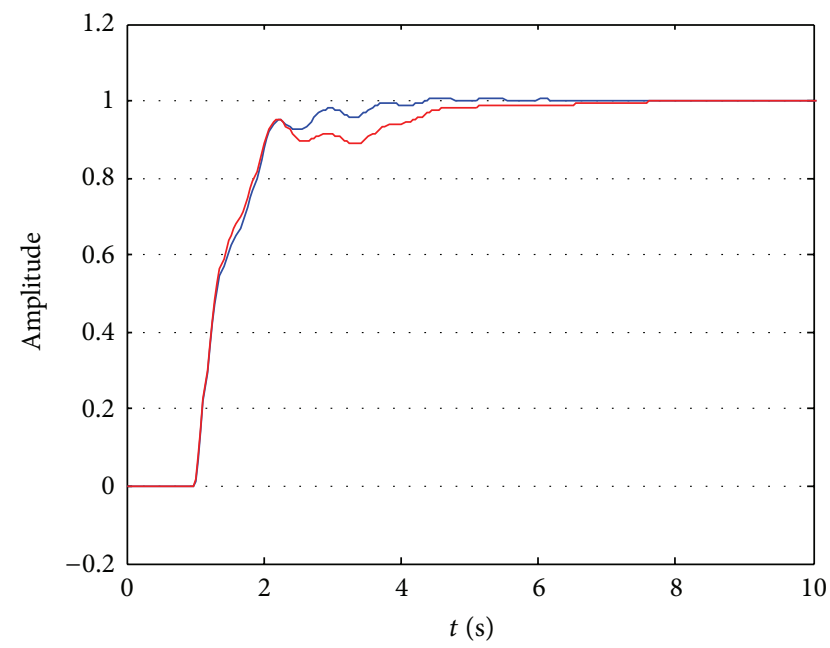

(b) $90 \%$ load-disturbance

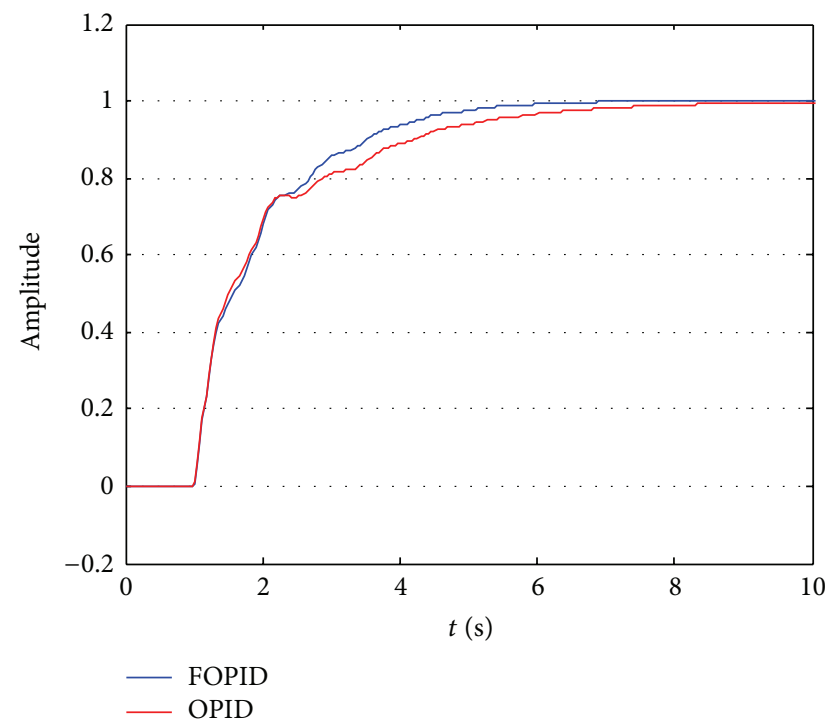

(d) $70 \%$ load-disturbance

FIGURE 11: Load-disturbance response of $G_{3}(s)$.

controller. As a whole, the simulation results clearly show the performance advantage of the proposed FOPID controller based on numerical inverse Laplace transform algorithms when compared with the conventional OPID controller for oscillatory fractional time-delay systems.

4.3. Comparative Performance of Different Controllers for High-Order Complex Fractional System with Time Delay. In this section, we had also used another complex example to verify the effectiveness of the proposed controller. It is a highorder complex fractional-order delay system whose transfer function is

$$
G_{4}(s)=\frac{e^{-s}}{s^{2.6}+2.2 s^{1.5}+2.9 s^{1.3}+3.32 s^{0.9}+1} .
$$

The proposed optimal FOPID controller and PID controller are achieved as

$$
\begin{aligned}
& G_{f_{c}}=3.9762+\frac{0.5313}{s^{1.0399}}+2.7176 s^{1.0049}, \\
& G_{c}=4.3510+2.96 s+\frac{0.7194}{s} .
\end{aligned}
$$

The set-point tracking control performance comparison and the disturbance rejection responses of $\pm 10 \%, \pm 30 \%$ were shown in Figures 12 and 13.

The results were similar to those of $G_{3}(s)$. The overshoot and accommodation time of FOPID controller are still more competitive. For the robustness measurements in Figure 13, FOPID controllers still perform well with satisfactory overshoots, rising time, and accommodation time, and the performance of OPID is also acceptable from the point of comparison. 


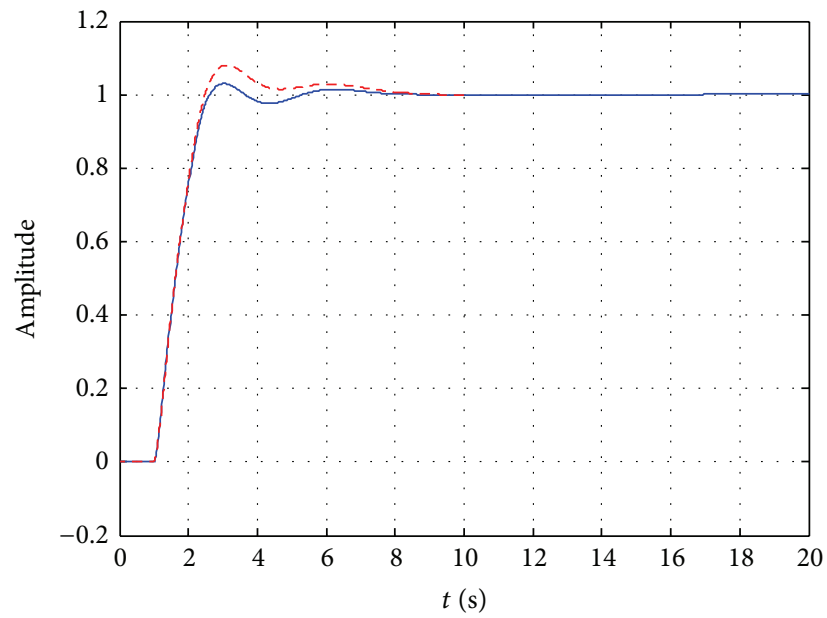

- FOPID

--- OPID

FIGURE 12: Set-pointing tracking control performance comparison of $G_{4}(s)$.

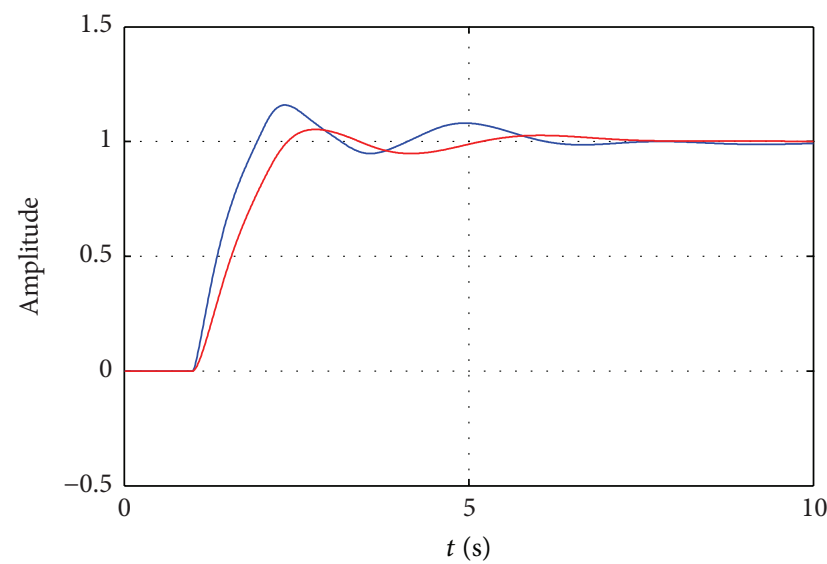

(a) $110 \%$ load-disturbance

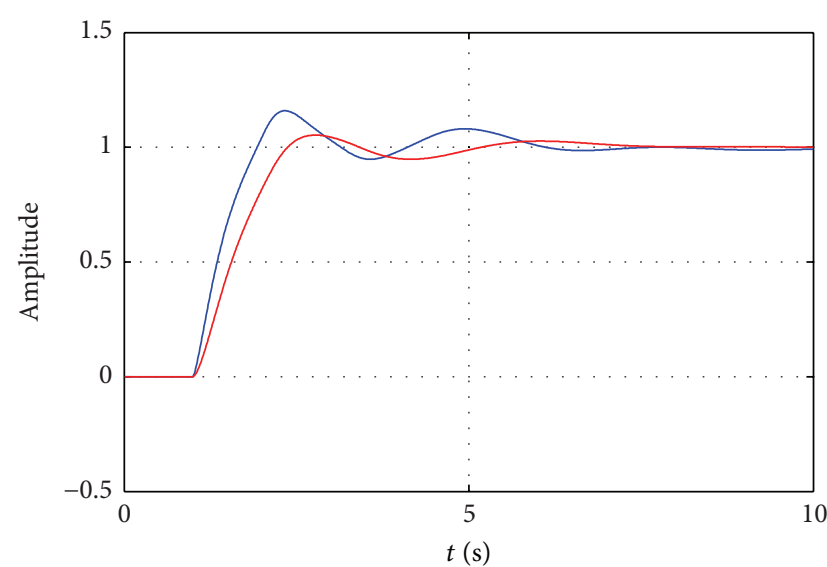

(c) $130 \%$ load-disturbance

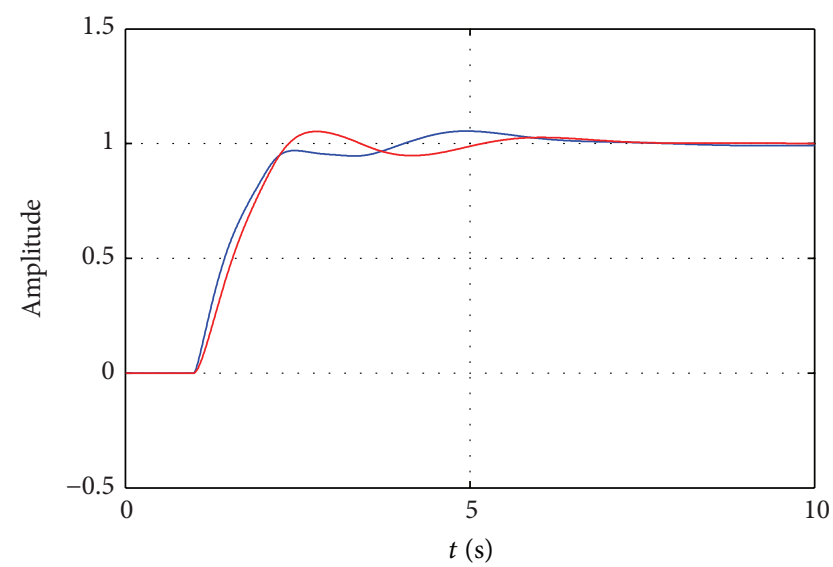

(b) $90 \%$ load-disturbance

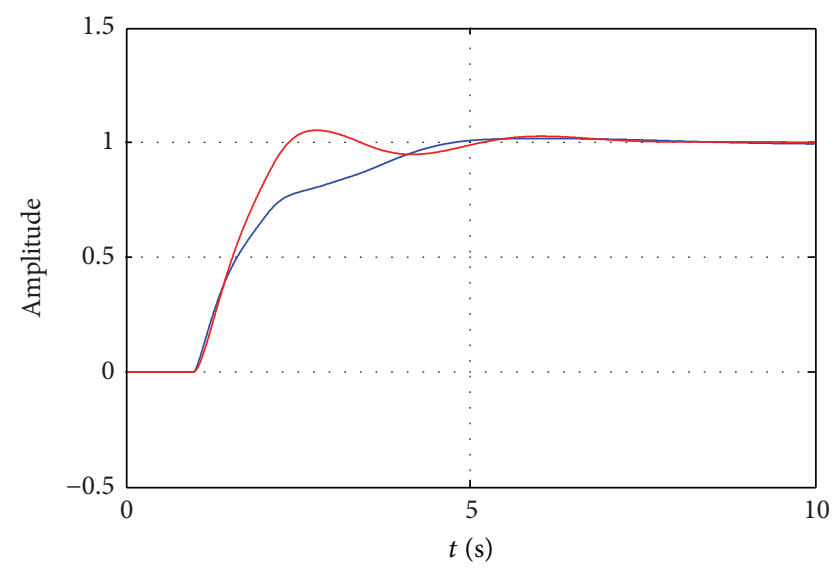

(d) $70 \%$ load-disturbance

FIGURE 13: Load-disturbance response of $G_{4}(s)$. 


\section{Conclusion}

In this paper, we presented a study of fractional $P I^{\lambda} D^{\mu}$ controller for oscillatory fractional time-delay systems based on the numerical invert Laplace transform algorithm INVLAP. The algorithm has been applied to solve the problem caused by the difficulties of searching for analytical solution of complicated fractional-order differential equations. Simulation results of comparisons of the proposed FOPID controllers and conventional OPID controllers applied on the example typical systems show that the closed-loop system with FOPID controller can achieve more satisfactory dynamic performance with faster and smoother closed-loop time responses and robustness coping with both set-point tracking and loaddisturbance rejection problems. And this method could be extended to be used on various complicated fractional-order dynamic models.

\section{Conflict of Interests}

The authors declare that there is no conflict of interests regarding the publication of this paper.

\section{Acknowledgments}

The work is supported by National Nature Science Foundation under Grant 61174145 and the Fundamental Research Funds for the Central Universities under Grant N110804005.

\section{References}

[1] A. Oustaloup, B. Mathieu, and P. Lanusse, "The CRONE control of resonant plants: application to a flexible transmission," European Journal of Control, vol. 1, no. 2, pp. 113-121, 1995.

[2] D. Matignon, "Stability results for fractional differential equations with applications to control processing," in Computational Engineering in Systems Applications, vol. 2, pp. 963-968, 1996.

[3] D. Matignon, "Some results on control ability and observability of finite-demensional fractional differential systems," in Proceedings of the Computational Engineering in Systems and Application Multi Conference, pp. 952-956, Lille, France, July 1996.

[4] I. Podlubny, Fractional Differential Equations: An Introduction to Fractional Derivatives, Fractional Differential Equations, to Methods of Their Solution and Some of Their Applications, Academic Press, 1998.

[5] C. A. Monje, Y. Chen, B. M. Vinagre et al., Fractional-Order Systems and Controls: Fundamentals and Applications, Springer, New York, NY, USA, 2010.

[6] Y. Q. Chen, B. M. Vinagre, and I. Podlubny, "Continued fraction expansion approaches to discretizing fractional order derivatives-an expository review," Nonlinear Dynamics, vol. 38, no. 1-4, pp. 155-170, 2004.

[7] R. L. Magin, Fractional Calculus in Bioengineering, Begell House Publishers, Redding, Calif, USA, 2006.

[8] H. Sheng, H. G. Sun, C. Coopmans, Y. Q. Chen, and G. W. Bohannan, "A Physical experimental study of variable-order fractional integrator and differentiator," The European Physical Journal: Special Topics, vol. 193, no. 1, pp. 93-104, 2011.
[9] H. Sheng and Y. Chen, "FARIMA with stable innovations model of Great Salt Lake elevation time series," Signal Processing, vol. 91, no. 3, pp. 553-561, 2011.

[10] C. A. Monje, B. M. Vinagre, V. Feliu, and Y. Chen, “Tuning and auto-tuning of fractional order controllers for industry applications," Control Engineering Practice, vol. 16, no. 7, pp. 798-812, 2008.

[11] D. Valério and J. S. da Costa, "Tuning of fractional PID controllers with Ziegler-Nichols-type rules," Signal Processing, vol. 86, no. 10, pp. 2771-2784, 2006.

[12] C. A. Monje, A. J. Calderon, B. M. Vinagre, Y. Chen, and V. Feliu, "On fractional PI $\lambda$ controllers: some tuning rules for robustness to plant uncertainties," Nonlinear Dynamics, vol. 38, no. 1-4, pp. 369-381, 2004.

[13] N. Sadati, M. Zamani, and P. Mohajerin, "Optimum design of fractional order PID for MIMO and SISO systems using particle swarm optimization techniques," in Proceedings of the 4th IEEE International Conference on Mechatronics (ICM '07), pp. 1-6, May 2007.

[14] S. A. Chen, Y. Y. Ou, and M. M. Gromiha, “Topology prediction of $\alpha$-helical and $\beta$-barrel transmembrane proteins using RBF networks," in Advanced Intelligent Computing Theories and Applications, vol. 6215 of Lecture Notes in Computer Science, pp. 642-649, Springer, Berlin, Germany, 2010.

[15] S. Das and I. Pan, "Performance comparison of optimal fractional order hybrid fuzzy PID controllers for handling oscillatory fractional order processes with dead time," ISA Transactions, vol. 52, no. 4, pp. 550-566, 2013.

[16] I. Pan, S. Das, and A. Gupta, "Tuning of an optimal fuzzy PID controller with stochastic algorithms for networked control systems with random time delay," ISA Transactions, vol. 50, no. 1, pp. 28-36, 2011.

[17] H. Sheng, Y. Li, and Y. Q. Chen, "Application of numerical inverse Laplace transform algorithms in fractional calculus," Journal of the Franklin Institute, vol. 348, no. 2, pp. 315-330, 2011.

[18] T. Hosono, "Numerical inversion of Laplace transform and some applications to wave optics," Radio Science, vol. 16, no. 6, pp. 1015-1019, 1980.

[19] C. Hwang and Y.-C. Cheng, "A numerical algorithm for stability testing of fractional delay systems," Automatica, vol. 42, no. 5, pp. 825-831, 2006.

[20] J. Sabatier, O. P. Agrawal, and J. A. T. Machado, Eds., Advances in Fractional Calculus, Springer, Dordrecht, The Netherlands, 2007.

[21] J. Valsa and L. Brančik, "Approximate formulae for numerical inversion of laplace transforms," International Journal of Numerical Modelling: Electronic Networks, Devices and Fields, vol. 11, no. 3, pp. 153-166, 1998.

[22] I. Podlubny, "Fractional-order systems and $\mathrm{PI}^{\lambda} \mathrm{D}^{\mu}$-controllers," IEEE Transactions on Automatic Control, vol. 44, no. 1, pp. 208214, 1999.

[23] M. S. Tavazoei, "Notes on integral performance indices in fractional-order control systems," Journal of Process Control, vol. 20, no. 3, pp. 285-291, 2010.

[24] R. R. Barton and J. S. Ivey Jr., "Nelder-Mead simplex modifications for simulation optimization," Management Science, vol. 42, no. 7, pp. 954-973, 1996.

[25] D. Xue and Y. Q. Chen, Modeling, Analysis and Design of Control Systems with MATLAB and Simulink, World Scientific, Singapore, 2014. 


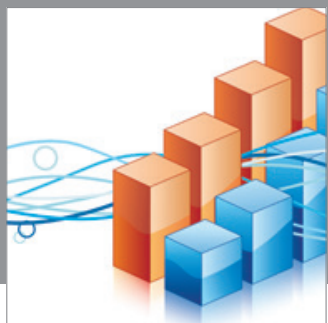

Advances in

Operations Research

mansans

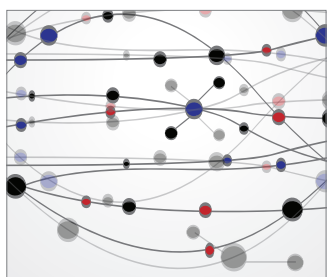

The Scientific World Journal
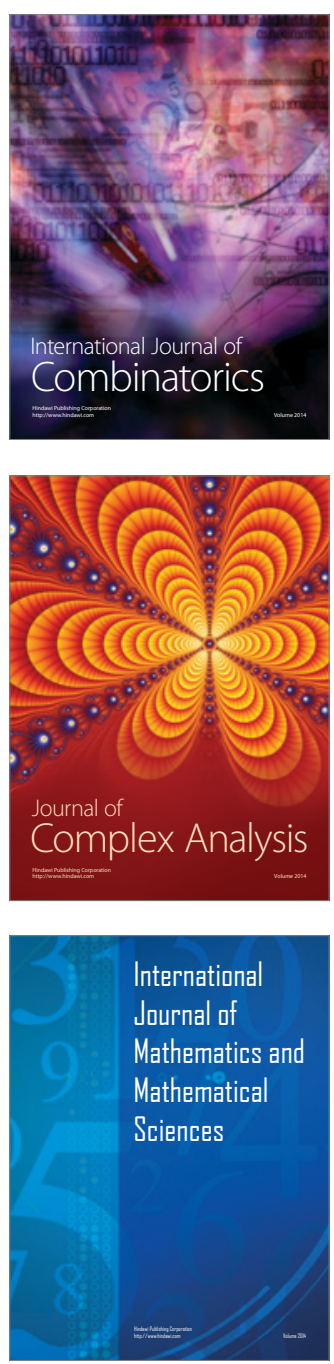
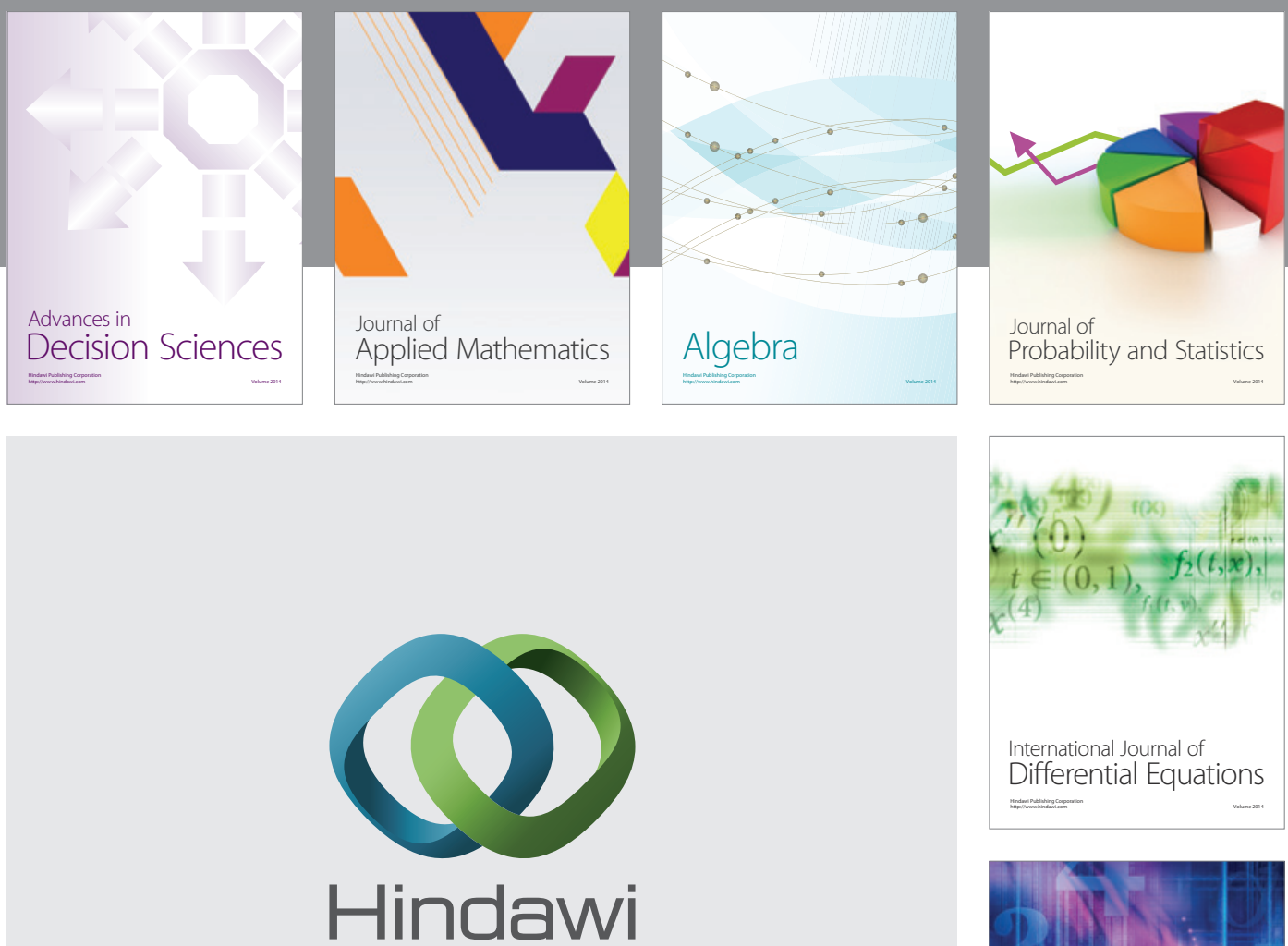

Submit your manuscripts at http://www.hindawi.com
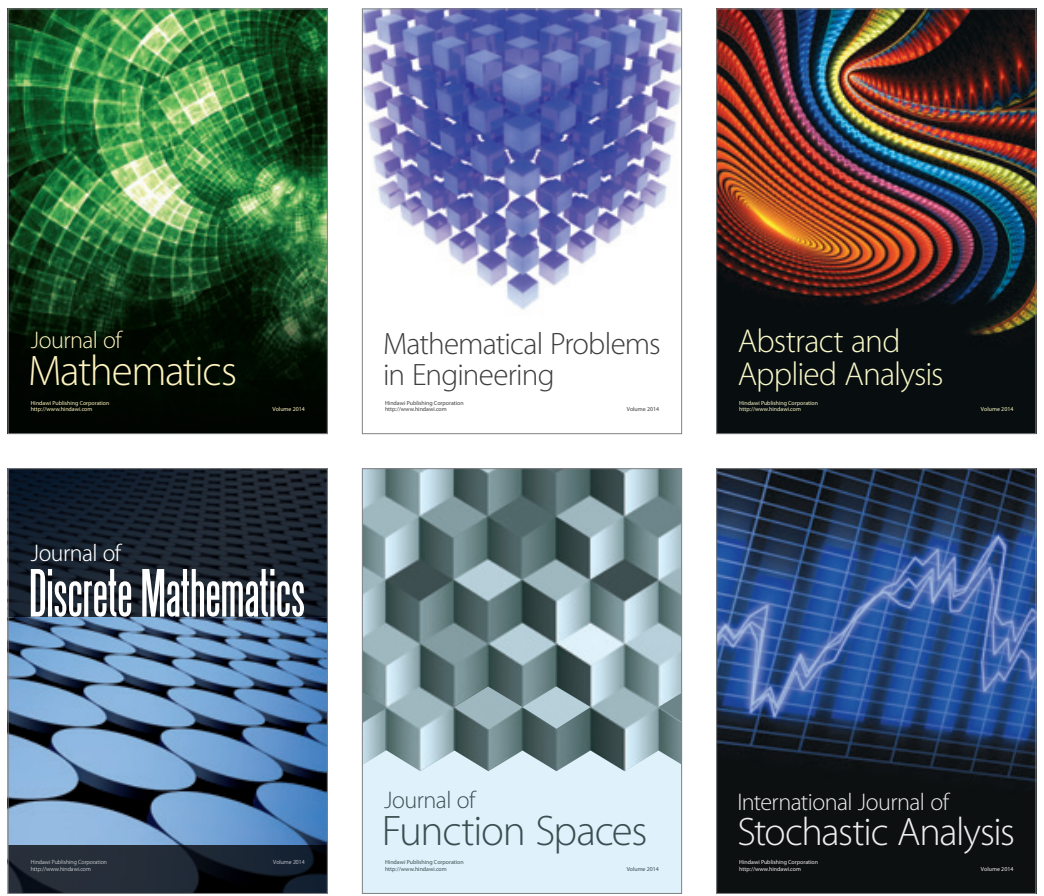

Journal of

Function Spaces

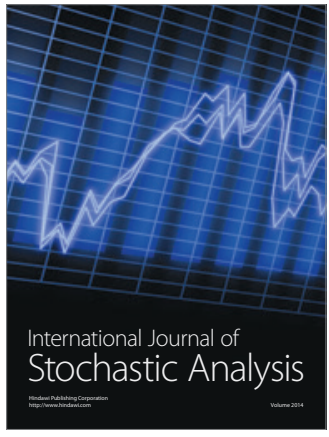

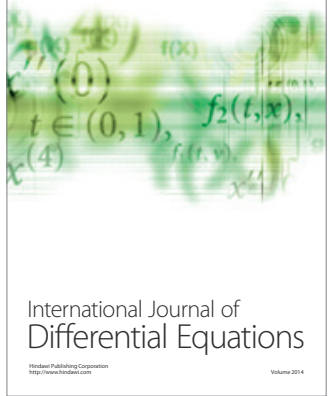
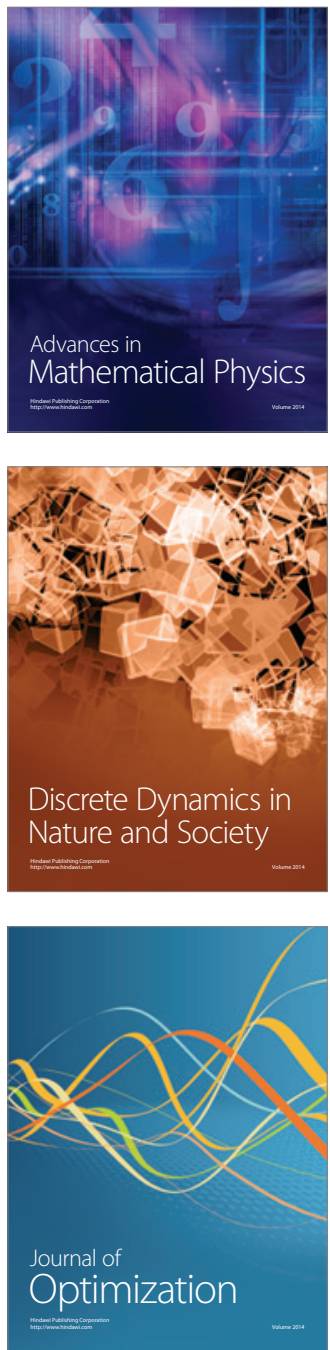